\title{
Anomalous dimensions of four-quark operators and renormalon structure of mesonic two-point correlators
}

\author{
Diogo Boito, ${ }^{a, b}$ Dirk Hornung ${ }^{c}$ and Matthias Jamin ${ }^{d}$ \\ ${ }^{a}$ Instituto de Física de São Carlos, Universidade de São Paulo, \\ CP 369, 13560-970, São Carlos, SP, Brazil \\ ${ }^{b}$ Instituto de Fúsica, Universidade de São Paulo, \\ Rua do Matão Travessa R, 187, 05508-090, São Paulo, SP, Brazil \\ ${ }^{c}$ Institut de Física d'Altes Energies (IFAE), The Barcelona Institute of Science and Technology, \\ Campus UAB, 08193 Bellaterra (Barcelona) Spain \\ ${ }^{d}$ Institució Catalana de Recerca i Estudis Avançats (ICREA), \\ Institut de Física d'Altes Energies (IFAE), The Barcelona Institute of Science and Technology, \\ Campus UAB, 08193 Bellaterra (Barcelona) Spain \\ E-mail: boito@ifsc.usp.br, dirk.hornung@uab.cat, jamin@ifae.es
}

ABSTRACT: In this work, we calculate leading-order anomalous dimension matrices for dimension-6 four-quark operators which appear in the operator product expansion of flavour non-diagonal and diagonal vector and axial-vector two-point correlation functions. The infrared renormalon structure corresponding to four-quark operators is reviewed and it is investigated how the eigenvalues of the anomalous dimension matrices influence the singular behaviour of the $u=3$ infrared renormalon pole. It is found that compared to the large- $\beta_{0}$ approximation where at most quadratic poles are present, in full QCD at $N_{f}=3$ the most singular pole is more than cubic with an exponent $\kappa \approx 3.2$.

KEYwords: Renormalization Group, QCD, Renormalization Regularization and Renormalons

ARXIV EPRINT: 1510.03812 


\section{Contents}

1 Introduction 1

2 Flavour non-diagonal vector and axial-vector correlators 3

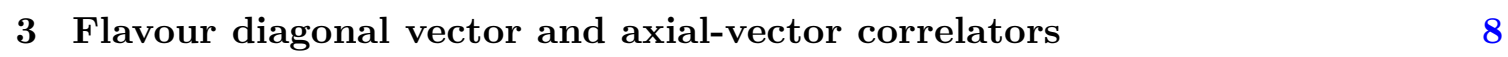

4 Renormalon structure of dimension-6 four-quark operators $\quad 10$

5 Conclusions $\quad 12$

$\begin{array}{ll}\text { A Anomalous dimensions of four-quark operators } & 13\end{array}$

$\begin{array}{ll}\text { B Eigenvectors of anomalous dimension matrices } & 15\end{array}$

\section{Introduction}

The perturbative expansion in QCD is known to lead to a divergent series which is at best asymptotic. The asymptotic behaviour of the perturbative series manifests itself in the appearance of singularities for its Borel transform which lie on the negative or positive real axis in the Borel variable. Those singularities, connected with renormalisation of the theory, are termed renormalons [1,2]. More specifically, singularities related to the shortdistance behaviour of the theory lie on the negative real axis and are called ultraviolet (UV) renormalons. Those related to long-distance physics appear on the positive real Borel axis and are termed infrared (IR) renormalons.

The presence of IR renormalon poles leads to ambiguities in the definition of the full function which is related to the perturbative series, because the Borel resummation (inverse Borel transform) entails to perform an integral over the positive real Borel axis which naively is not well defined. The ambiguities in the definition of the Borel integral are exponentially small terms in the QCD coupling, $\alpha_{s}$. Associated with them is the appearance of higher-dimensional operator corrections, the so-called QCD condensates, such that the full function is unambiguous. The operators that display renormalon ambiguities are a subset of those that arise in the framework of the operator product expansion (OPE).

The renormalisation group ( $\mathrm{RG}$ ) allows one to relate the exponent of a given renormalon pole to the leading-order anomalous dimension of the associated operator. Therefore, knowledge about the anomalous dimensions of operators entering the OPE expansion of a correlator can be translated into knowledge of the Borel transform of its purely perturbative contribution. The position and strength of poles can, in principle, be predicted in this way while the residua, which are non-perturbative, cannot. This partial knowledge of the Borel transformed perturbative series can be exploited as a way to gain understanding 
about higher orders, yet unknown from loop computations. In particular, in the case of the QCD description of hadronic $\tau$ decays [3], models of the Borel transform have been used in order to assess - among other things - the virtues of different RG improvement prescriptions $[4,5]$. It is with this type of application in mind that we revisit the computation of anomalous dimensions of four-quark operators.

Limiting ourselves to correlation functions of vector or axial-vector currents with respect to the QCD vacuum, the IR renormalon pole on the positive real axis closest to the origin of the Borel plane is associated to the vacuum matrix element of one dimension-four operator, the gluon condensate. The next-to-closest singularity then is found to correspond to the dimension- 6 triple gluon condensate and a set of dimension- 6 four-quark condensates. It is these latter four-quark condensates that we investigate in more detail in the present work.

The central aim of the present study is to provide the leading-order anomalous dimension matrices corresponding to the dimension- 6 four-quark operators that emerge in the OPE of two-point correlation functions of flavour non-diagonal, as well as flavour diagonal, mesonic vector and axial-vector currents. Those anomalous dimensions contain information about the structure of the related IR renormalon poles. The computation of leading-order anomalous dimensions of four-fermion operators is fairly standard [6], and in ref. [7], results were presented for a complete set of spin-0 four-quark operators without derivatives in the case of three active light quark flavours, $N_{f}=3$.

As a matter of principle, all findings presented in this work are derivable from the results of ref. [7] through operator relations, valid in four space-time dimensions. However, the results in [7] (and also [6]), were only given for a number of quark colours $N_{c}=3$, and here we intend to provide results at arbitrary $N_{c}$. Furthermore, in order to be able to connect to the large- $N_{f}$, or the related large- $\beta_{0}$ limit, of QCD [2], in contrast to ref. [7], explicit $N_{f}$ dependencies will be kept. To this end, we therefore followed two alternative routes: first, to recalculate ref. [7] at arbitrary $N_{c}$, which is discussed in appendix A, and then derive the results below from the mentioned operator relations. And second, to compute the anomalous dimensions directly for the operators appearing in the correlation functions. Both approaches lead to agreeing results and also at $N_{c}=N_{f}=3$, the anomalous dimension matrices of appendix A coincide with [7]. Furthermore, in some test cases that we checked, also full agreement with the results of ref. [6] was found.

In summary, the material presented in this work is organised as follows: first, in section 2, we review the next-to-leading order results on the dimension- 6 four-quark operator contributions to the flavour non-diagonal vector and axial-vector correlation functions available in the literature $[8,9]$. For the set of appearing operators, we calculate the leading-order anomalous dimension matrices. In order to obtain a set which closes under renormalisation, three dimension- 6 four-quark operators of penguin type have to be included. Analogous results are provided in section 3 for the flavour diagonal vector and axial-vector correlators.

Next, we construct the operator combinations for which the leading-order anomalous dimension matrices are diagonal and compute the corresponding eigenvalues. These eigenvalues are ingredients for the renormalon structure of the perturbative series which is 
related to the four-quark operators and discussed in section 4. Along these lines, we determine the singular behaviour of the relevant IR renormalon poles. Finally, in section 5, we end with a summary and conclusions, and appendix A provides the extension of the work of ref. [7] to an arbitrary number of colours $N_{c}$.

\section{Flavour non-diagonal vector and axial-vector correlators}

We begin by investigating the dimension- 6 OPE contributions to the two-point correlation functions of flavour non-diagonal vector and axial-vector currents $j_{\mu}^{V}(x)=\left(\bar{u} \gamma_{\mu} d\right)(x)$ and $j_{\mu}^{A}(x)=\left(\bar{u} \gamma_{\mu} \gamma_{5} d\right)(x)$ which are relevant for QCD analyses of hadronic $\tau$ decays [3] and correspond to the charged $\rho$ and $A_{1}$ mesons. For simplicity, massless light quarks will be assumed in which case the correlators take the form

$$
\Pi_{\mu \nu}^{V / A}(q) \equiv i \int \mathrm{d} x \mathrm{e}^{i q x}\left\langle\Omega\left|T\left\{j_{\mu}^{V / A}(x) j_{\nu}^{V / A}(0)^{\dagger}\right\}\right| \Omega\right\rangle=\left(q_{\mu} q_{\nu}-g_{\mu \nu} q^{2}\right) \Pi^{V / A}\left(q^{2}\right) .
$$

Here, $|\Omega\rangle$ denotes the full QCD vacuum and the second identity follows because in the massless limit vector and axial-vector currents are conserved.

In the framework of the OPE, the scalar functions $\Pi^{V / A}$ permit an expansion in powers of $1 / Q^{2}$ with $Q^{2} \equiv-q^{2}>0$ being in the Euclidean region,

$$
\Pi^{V / A}\left(Q^{2}\right)=C_{0}\left(Q^{2}\right)+C_{4}\left(Q^{2}\right) \frac{\left\langle O_{4}\right\rangle}{Q^{4}}+C_{6}^{V / A}\left(Q^{2}\right) \frac{\left\langle O_{6}\right\rangle}{Q^{6}}+\ldots
$$

In writing eq. (2.2), for simplicity, we have suppressed the vacuum state. In the OPE, only the coefficient functions $C_{i}^{V / A}$ depend on the momentum, while the operators $O_{i}$ are local. Both, coefficient functions and operators depend, however, on the renormalisation scale $\mu$ which is not shown explicitly. Furthermore, for flavour non-diagonal currents the purely perturbative contribution $C_{0}\left(Q^{2}\right)$ is the same for vector and axial vector. ${ }^{1}$ In the massless case, this also remains true for the dimension- 4 contribution, which then only consists of the gluon condensate $\left\langle G_{\mu \nu}^{a} G^{a \mu \nu}\right\rangle$.

Our main concern in this work will be the dimension- 6 term which receives contributions from the three-gluon condensate $\left\langle g^{3} f_{a b c} G_{\mu \nu}^{a} G^{b \nu}{ }_{\lambda} G^{c \lambda \mu}\right\rangle$ and four-quark condensates. As the three-gluon condensate does not arise at leading order, below we concentrate only on the four-quark condensates. Their contribution to $\Pi^{V / A}\left(Q^{2}\right)$ has been computed at the next-to-leading order in refs. $[8,9]$. For our following discussion, it will be convenient to present the corresponding results for $V-A$ and $V+A$ correlation functions, because in the former case the so-called penguin operator contributions cancel. For $N_{f}=3$ light quark flavours and at $N_{c}=3$, one then finds

$$
C_{6}^{V-A}\left(Q^{2}\right)\left\langle O_{6}\right\rangle=4 \pi^{2} a_{s}\left\{\left[2+\left(\frac{25}{6}-L\right) a_{s}\right]\left\langle Q_{-}^{o}\right\rangle-\left(\frac{11}{18}-\frac{2}{3} L\right) a_{s}\left\langle Q_{-}^{s}\right\rangle\right\}
$$

\footnotetext{
${ }^{1}$ For correlators of flavour-diagonal currents, which will be discussed below, this is not the case.
} 
and

$$
\begin{aligned}
C_{6}^{V+A}\left(Q^{2}\right)\left\langle O_{6}\right\rangle=-4 \pi^{2} a_{s}\{ & {\left[2+\left(\frac{155}{24}-\frac{7}{2} L\right) a_{s}\right]\left\langle Q_{+}^{o}\right\rangle+\left(\frac{11}{18}-\frac{2}{3} L\right) a_{s}\left\langle Q_{+}^{s}\right\rangle } \\
+\left[\frac{4}{9}\right. & \left.+\left(\frac{37}{36}-\frac{95}{162} L\right) a_{s}\right]\left\langle Q_{3}\right\rangle\left(\frac{35}{108}-\frac{5}{18} L\right) a_{s}\left\langle Q_{4}\right\rangle+ \\
& +\left(\frac{14}{81}-\frac{4}{27} L\right) a_{s}\left\langle Q_{6}\right\rangle-\left(\frac{2}{81}+\frac{4}{27} L\right) a_{s}\left\langle Q_{7}\right\rangle .
\end{aligned}
$$

Here, $a_{s} \equiv \alpha_{s} / \pi, L \equiv \ln Q^{2} / \mu^{2}$ and the constant terms of order $a_{s}^{2}$ correspond to the choice of an anti-commuting $\gamma_{5}$ in $D$ space-time dimensions which can be made consistent as long as no traces with an odd number of $\gamma_{5}$ 's arise in the calculation [8].

The appearing four-quark operators are a subset which belong to the complete basis that below will be required for their one-loop renormalisation:

$$
\begin{array}{rlrl}
Q_{V}^{o} & =\left(\bar{u} \gamma_{\mu} t^{a} d \bar{d} \gamma^{\mu} t^{a} u\right), & Q_{A}^{o}=\left(\bar{u} \gamma_{\mu} \gamma_{5} t^{a} d \bar{d} \gamma^{\mu} \gamma_{5} t^{a} u\right) \\
Q_{V}^{s} & =\left(\bar{u} \gamma_{\mu} d \bar{d} \gamma^{\mu} u\right), & Q_{A}^{s}=\left(\bar{u} \gamma_{\mu} \gamma_{5} d \bar{d} \gamma^{\mu} \gamma_{5} u\right) \\
Q_{3} & \equiv\left(\bar{u} \gamma_{\mu} t^{a} u+\bar{d} \gamma_{\mu} t^{a} d\right) \sum_{q=u, d, s}\left(\bar{q} \gamma^{\mu} t^{a} q\right), & \\
Q_{4} & \equiv\left(\bar{u} \gamma_{\mu} \gamma_{5} t^{a} u+\bar{d} \gamma_{\mu} \gamma_{5} t^{a} d\right) \sum_{q=u, d, s}\left(\bar{q} \gamma^{\mu} \gamma_{5} t^{a} q\right), & \\
Q_{5} & \equiv\left(\bar{u} \gamma_{\mu} u+\bar{d} \gamma_{\mu} d\right) \sum_{q=u, d, s}\left(\bar{q} \gamma^{\mu} q\right) \\
Q_{6} & \equiv\left(\bar{u} \gamma_{\mu} \gamma_{5} u+\bar{d} \gamma_{\mu} \gamma_{5} d\right) \sum_{q=u, d, s}\left(\bar{q} \gamma^{\mu} \gamma_{5} q\right), \\
Q_{7} & \equiv \sum_{q=u, d, s}\left(\bar{q} \gamma_{\mu} t^{a} q\right) \sum_{q^{\prime}=u, d, s}\left(\bar{q}^{\prime} \gamma^{\mu} t^{a} q^{\prime}\right) \\
Q_{8} & \equiv \sum_{q=u, d, s}\left(\bar{q} \gamma_{\mu} \gamma_{5} t^{a} q\right) \sum_{q^{\prime}=u, d, s}\left(\bar{q}^{\prime} \gamma^{\mu} \gamma_{5} t^{a} q^{\prime}\right), \\
Q_{9} & \equiv \sum_{q=u, d, s}\left(\bar{q} \gamma_{\mu} q\right) \sum_{q^{\prime}=u, d, s}\left(\bar{q}^{\prime} \gamma^{\mu} q^{\prime}\right) \\
Q_{10} & \equiv \sum_{q=u, d, s}\left(\bar{q} \gamma_{\mu} \gamma_{5} q\right) \sum_{q^{\prime}=u, d, s}\left(\bar{q}^{\prime} \gamma^{\mu} \gamma_{5} q^{\prime}\right) .
\end{array}
$$

The operators $Q_{V / A}^{o}$ and $Q_{V / A}^{s}$ are usually termed current-current operators and $Q_{3}$ to $Q_{10}$ penguin operators. In addition, we define the four current-current operators which appear in eqs. (2.3) and (2.4).

$$
Q_{ \pm}^{o} \equiv Q_{V}^{o} \pm Q_{A}^{o}, \quad Q_{ \pm}^{s} \equiv Q_{V}^{s} \pm Q_{A}^{s}
$$

Next, we investigate the scale dependence of a general term $R_{O}$ in the OPE, corresponding to a set of operators $\vec{O}$ with equal dimension,

$$
R_{O}=\vec{C}^{T}(\mu)\langle\vec{O}(\mu)\rangle
$$


where now the renormalisation scale $\mu$ is displayed explicitly and the potential dependence on other dimensionful parameters is implicit. For vector and axial-vector currents, the renormalisation scale dependence of the correlator only arises from the purely perturbative contribution. Hence, $R_{O}$ should not depend on $\mu$, and it immediately follows that

$$
\left[\mu \frac{\mathrm{d}}{\mathrm{d} \mu} \vec{C}^{T}(\mu)\right]\langle\vec{O}(\mu)\rangle=-\vec{C}^{T}(\mu)\left[\mu \frac{\mathrm{d}}{\mathrm{d} \mu}\langle\vec{O}(\mu)\rangle\right] .
$$

The anomalous dimension matrix $\hat{\gamma}_{O}$ of the operator matrix elements can be defined by

$$
-\mu \frac{\mathrm{d}}{\mathrm{d} \mu}\langle\vec{O}(\mu)\rangle \equiv \hat{\gamma}_{O}\left(a_{\mu}\right)\langle\vec{O}(\mu)\rangle,
$$

with $a_{\mu} \equiv a_{s}(\mu)$. If the bare and renormalised operator matrix elements are related by

$$
\left\langle\vec{O}^{B}\right\rangle \equiv \hat{Z}_{O}(\mu)\langle\vec{O}(\mu)\rangle,
$$

it follows that the anomalous dimension matrix can be computed from the renormalisation matrix $\hat{Z}_{O}(\mu)$ via

$$
\hat{\gamma}_{O}\left(a_{\mu}\right)=\hat{Z}_{O}^{-1}(\mu) \mu \frac{\mathrm{d}}{\mathrm{d} \mu} \hat{Z}_{O}(\mu) .
$$

Plugging eq. (2.18) into the RGE for $R$, eq. (2.17), one obtains an RGE that has to be satisfied by the coefficient functions $\vec{C}(\mu)$,

$$
\mu \frac{\mathrm{d}}{\mathrm{d} \mu} \vec{C}(\mu)=\hat{\gamma}_{O}^{T}\left(a_{\mu}\right) \vec{C}(\mu) .
$$

This equation shall be checked for the coefficient functions of the dimension- 6 operators in eqs. (2.3) and (2.4). Furthermore, below it will be convenient to consider the anomalous dimension matrix in a linearly transformed basis. If the transformed basis $\left\langle\vec{O}^{\prime}(\mu)\right\rangle$ of operator matrix elements is defined by

$$
\left\langle\vec{O}^{\prime}(\mu)\right\rangle \equiv \hat{T}\langle\vec{O}(\mu)\rangle
$$

the corresponding transformed anomalous dimension matrix takes the form

$$
\hat{\gamma}_{O^{\prime}}=\hat{T} \hat{\gamma}_{O} \hat{T}^{-1} \text {. }
$$

The calculation of one-loop anomalous dimension matrices for dimension-6 four-quark operators is fairly standard and details can for example be found in ref. [6]. We have performed the actual computation in two ways: firstly, by explicit calculation of the diagrams of figure 1, and secondly, by relating the appearing operators to the complete basis of dimension- 6 four-quark operators without derivatives in the case of three quark flavours that had been employed in ref. [7]. Further details on the second approach can be found in appendix A. The computation has been performed in a general covariant gauge in order to explicitly verify gauge invariance.

Expanding the anomalous dimension matrix in a power series in $a_{s}$,

$$
\hat{\gamma}_{O}\left(a_{s}\right)=a_{s} \hat{\gamma}_{O}^{(1)}+a_{s}^{2} \hat{\gamma}_{O}^{(2)}+\ldots,
$$

the leading-order anomalous dimension matrix corresponding to the operators $Q_{-} \equiv$ $\left(Q_{-}^{o}, Q_{-}^{s}\right)$ appearing in eq. (2.3) is found to be

$$
\hat{\gamma}_{Q_{-}}^{(1)}=\left(\begin{array}{cc}
-\frac{3 N_{c}}{2}+\frac{3}{N_{c}} & -\frac{3 C_{F}}{2 N_{c}} \\
-3 & 0
\end{array}\right) .
$$




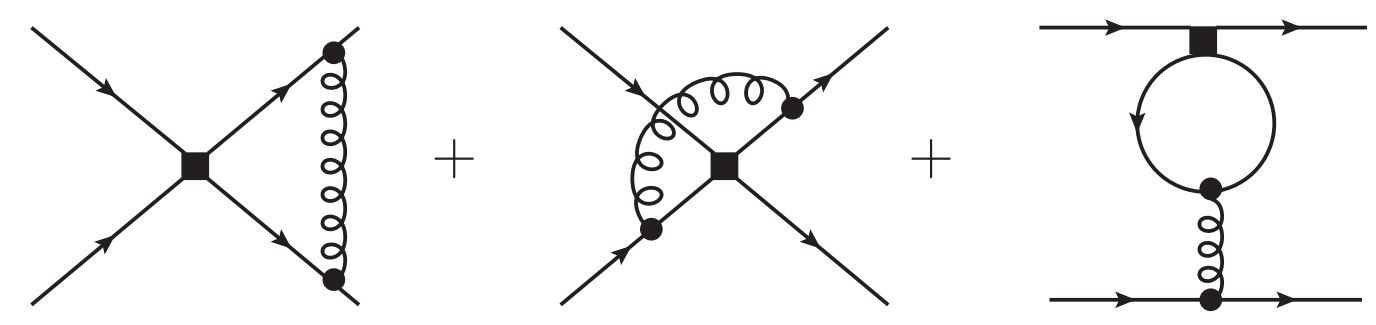

Figure 1. Exemplary one-loop current-current and penguin diagrams that have to be calculated in the process of obtaining the leading-order anomalous dimension matrix of four-quark operators. Quark self-energy diagrams are not displayed.

At this order, the set of two operators is closed under renormalisation, meaning that no additional operators are generated through the diagrams that have to be computed. ${ }^{2}$ Employing $\hat{\gamma}_{Q_{-}}^{(1)}$ and the coefficient function $C_{6}^{V-A}\left(Q^{2}\right)$ of eq. (2.3), it is a simple matter to confirm that the RGE (2.21) is indeed satisfied.

Likewise, the anomalous dimension matrix for the operators appearing in the $V+A$ case of eq. (2.4) can be calculated. Here, three additional operators have to be added in the course of renormalisation, and at the leading order a closed set can be chosen as $Q_{+} \equiv\left(Q_{+}^{o}, Q_{+}^{s}, Q_{3}, Q_{4}, Q_{6}, Q_{7}, Q_{8}, Q_{9}, Q_{10}\right)$. In general, also the operator $Q_{5}$ of the basis presented above arises. However, in four dimensions, one operator in the full set is redundant and can be expressed through the others by means of Fierz transformations. Since $Q_{5}$ does not appear in the OPE expression (2.4), we have rewritten it through the remaining operators (see eq. (A.9)). The anomalous dimension matrix then takes the form

$$
\hat{\gamma}_{Q_{+}}^{(1)}=\left(\begin{array}{cccc}
-\frac{3}{N_{c}} & \frac{3 C_{F}}{2 N_{c}} & -\frac{1}{3 N_{c}} & 0 \\
3 & 0 & \frac{2}{3} & 0 \\
0 & 0 & \frac{N_{f}}{3}-\frac{3 N_{c}}{4}-\frac{1}{3 N_{c}} & \frac{3 N_{c}}{4}-\frac{3}{N_{c}} \\
\frac{3}{2}+\frac{3}{2 N_{c}} & -\frac{3 C_{F}}{2 N_{c}} & \frac{3 N_{c}}{4}+\frac{3}{2}-\frac{11}{6 N_{c}} & -\frac{3 N_{c}}{4}+\frac{3}{2}+\frac{3}{2 N c} \\
0 & 0 & \frac{11}{3} & 0 \\
0 & 0 & 0 & 0 \\
0 & 0 & 0 & 0 \\
0 & 0 & 0 & 0 \\
0 & 0 & 0 & 0
\end{array}\right.
$$

$$
\left.\begin{array}{ccccc}
0 & 0 & 0 & 0 & 0 \\
0 & 0 & 0 & 0 & 0 \\
\frac{3 C_{F}}{2 N_{c}} & \frac{2}{3} & 0 & 0 & 0 \\
-\frac{3 C_{F}}{2 N_{c}} & -\frac{3}{4}-\frac{3}{4 N_{c}} & -\frac{3}{4}-\frac{3}{4 N_{c}} & \frac{3 C_{F}}{4 N_{c}} & \frac{3 C_{F}}{4 N_{c}} \\
0 & 0 & 0 & 0 & 0 \\
0 & \frac{2 N_{f}}{3}-\frac{3 N_{c}}{4}-\frac{1}{3 N_{c}} & \frac{3 N_{c}}{4}-\frac{3}{N_{c}} & 0 & \frac{3 C_{F}}{2 N_{c}} \\
0 & \frac{3 N_{c}}{4}-\frac{10}{3 N_{c}} & -\frac{3 N_{c}}{4} & \frac{3 C_{F}}{2 N_{c}} & 0 \\
0 & \frac{2}{3} & 3 & 0 & 0 \\
0 & \frac{11}{3} & 0 & 0 & 0
\end{array}\right) .
$$

In ref. [10], and section 3.2.3 of ref. [2], the $\mathrm{SU}(3)$ flavour-singlet operators $Q_{7}$ to $Q_{10}$ arose in an investigation of the structure of the leading UV renormalon at $u=-1$ for the vector correlator. Though not directly related to our study, the relevant leading-order anomalous

\footnotetext{
${ }^{2}$ This is only true when working with a strictly anti-commuting $\gamma_{5}$, and projecting out evanescent operators, which, however, is admissible at the leading order. Further discussion can be found in ref. [8].
} 
dimensions correspond to the $4 \times 4$ sub-matrix for $Q_{7}$ to $Q_{10}$, and comparing to the results of refs. [2, 10], we find complete agreement. ${ }^{3}$ Regarding the RGE, again, it is straightforward to verify that by using $\hat{\gamma}_{Q_{+}}^{(1)}$ and the coefficient function of eq. (2.4), the RGE (2.21) is satisfied to leading order.

For the subsequent discussion, it will be convenient to consider a basis of four-quark operators in which the leading-order anomalous dimension matrix is diagonal. From linear algebra it is well known that the anomalous dimension matrix $\hat{\gamma}_{O}^{(1)}$ can be diagonalised by a matrix $\hat{V}$, which as columns contains the eigenvectors of $\hat{\gamma}_{O}^{(1)}$, in the following fashion:

$$
\hat{\gamma}_{D}^{(1)}=\hat{V}^{-1} \hat{\gamma}_{O}^{(1)} \hat{V}
$$

The diagonal entries of $\hat{\gamma}_{D}^{(1)}$ then correspond to the eigenvalues of $\hat{\gamma}_{O}^{(1)}$. Furthermore, the operator basis with $\hat{\gamma}_{D}^{(1)}$ as the leading-order anomalous dimension matrix is given by $\hat{V}^{-1} \vec{O}$. Rewriting the term $R_{O}$ of (2.16) in the OPE,

$$
R_{O}=\vec{C}^{T}(\mu) \hat{V} \hat{V}^{-1}\langle\vec{O}(\mu)\rangle,
$$

the logarithms in $\vec{C}(\mu)$ can be resummed to leading order by solving the RGE (2.21), leading to

$$
R_{O}=\vec{C}^{T}(Q) \hat{V}\left[\left(\frac{a_{Q}}{a_{\mu}}\right)^{\vec{\gamma}_{D}^{(1)} / \beta_{1}}\right]_{D} \hat{V}^{-1}\langle\vec{O}(\mu)\rangle .
$$

The somewhat condensed notation in (2.29) should be read as follows: $\vec{\gamma}_{D}^{(1)}$ is a vector containing the eigenvalues of $\hat{\gamma}_{O}^{(1)}$ ordered according to the eigenvectors in $\hat{V}$. Then $[. .]_{D}$ is the diagonal matrix which contains as diagonal entries the ratios of $a_{s}$ to the power of elements in $\vec{\gamma}_{D}^{(1)} / \beta_{1}$. Finally, $\beta_{1}=11 N_{c} / 6-N_{f} / 3$ is the leading coefficient in the expansion of the QCD $\beta$-function

$$
-\mu \frac{\mathrm{d} a_{\mu}}{\mathrm{d} \mu} \equiv \beta\left(a_{\mu}\right)=\beta_{1} a_{\mu}^{2}+\beta_{2} a_{\mu}^{3}+\ldots .
$$

The generalisation of (2.29) to next-to-leading order is slightly non-trivial because anomalous dimension matrices at different couplings do not commute, but it can for example be found in refs. [12, 13].

Numerically, at $N_{c}=N_{f}=3$ the eigenvalues of the anomalous dimension matrices $\hat{\gamma}_{Q_{-}}^{(1)}$ and $\hat{\gamma}_{Q_{+}}^{(1)}$, ordered in increasing value, are found to be

$$
\begin{aligned}
& \vec{\gamma}_{D, Q_{-}}^{(1)}=(-4,0.5) \\
& \vec{\gamma}_{D, Q_{+}}^{(1)}=(-3.611,-3.387,-1.878,-1.494,0.538,0.567,1,1.340,1.703) .
\end{aligned}
$$

Besides the eigenvalue 1 , the entries in $\vec{\gamma}_{D, Q_{+}}^{(1)}$ are found as the roots of the two quartic polynomials $176-316 z-101 z^{2}+130 z^{3}+36 z^{4}$ and $88-122 z-91 z^{2}+47 z^{3}+18 z^{4}$. The corresponding eigenvectors have been collected in appendix B. Regarding the operator

\footnotetext{
${ }^{3}$ The same subset of anomalous dimensions was already considered in a previous attempt to investigate the $u=3$ renormalon structure [11].
} 
combinations $\hat{V}_{Q_{+}}^{-1} \vec{Q}_{+}$, it is found that four of them just include the operators $Q_{7}$ to $Q_{10}$, and a further combination misses the operator $Q_{6}$, while the remaining ones contain all contributing operators. The eigenvalues corresponding to the combinations only containing $Q_{7}$ to $Q_{10}$ (entries 2, 4, 6 and 9) agree with the result of table 1 in references [2, 10] where the same operators were considered to study the structure of the leading UV renormalon.

To conclude this section, we investigate the case of flavour SU(2). Then, the closed $(V+A)$ operator basis is given by $\bar{Q}_{+} \equiv\left(Q_{+}^{o}, Q_{+}^{s}, \bar{Q}_{3}, \bar{Q}_{4}, \bar{Q}_{6}\right)$, where in the penguin operators the strange quark is removed and hence the operators $\bar{Q}_{3}$ to $\bar{Q}_{6}$ are analogous to $Q_{7}$ to $Q_{10}$ with the sums just running over up and down quarks. The corresponding anomalous dimension matrix is found to be

$$
\hat{\gamma}_{\bar{Q}_{+}}(1)=\left(\begin{array}{ccccc}
-\frac{3}{N_{c}} & \frac{3 C_{F}}{2 N_{c}} & -\frac{1}{3 N_{c}} & 0 & 0 \\
3 & 0 & \frac{2}{3} & 0 & 0 \\
0 & 0 & \frac{2 N_{f}}{3}-\frac{3 N_{c}}{4}-\frac{1}{3 N_{c}} & \frac{3 N_{c}}{4}-\frac{3}{N_{c}} & \frac{3 C_{F}}{2 N_{c}} \\
3+\frac{3}{N_{c}} & -\frac{3 C_{F}}{N_{c}} & \frac{3 N_{c}}{4}+\frac{3}{2}-\frac{11}{6 N_{c}} & -\frac{3 N_{c}}{4}+\frac{3}{2}+\frac{3}{2 N c} & -\frac{3 C_{F}}{2 N_{c}} \\
0 & 0 & \frac{11}{3} & 0 & 0
\end{array}\right),
$$

with the eigenvalues

$$
\vec{\gamma}_{D, \bar{Q}_{+}}^{(1)}=(-3.521,-1.751,0.549,1,1.445)
$$

at $N_{c}=3$ and $N_{f}=2$. It is observed that the eigenvalues are slightly shifted with respect to the $\mathrm{SU}(3)$ case $(2.32)$, but span approximately the same range.

\section{Flavour diagonal vector and axial-vector correlators}

In this section, we now move to a discussion of dimension-6 OPE contributions to the flavour-diagonal vector and axial-vector currents. For definiteness, we consider the upquark case for which the corresponding currents are given by $j_{\mu}^{V}(x)=\left(\bar{u} \gamma_{\mu} u\right)(x)$ and $j_{\mu}^{A}(x)=\left(\bar{u} \gamma_{\mu} \gamma_{5} u\right)(x)$. The vector current for example is relevant in $e^{+} e^{-}$scattering to hadrons and carries the quantum numbers of the neutral $\rho$ meson.

To leading order, the contribution $C_{6}^{V \pm A}\left(Q^{2}\right)\left\langle O_{6}\right\rangle$ to the correlation function are the same as eqs. (2.3) and (2.4) with the current-current operators now being given by

$$
\begin{array}{rlrl}
Q_{V}^{o} & =\left(\bar{u} \gamma_{\mu} t^{a} u \bar{u} \gamma^{\mu} t^{a} u\right), & & Q_{A}^{o}=\left(\bar{u} \gamma_{\mu} \gamma_{5} t^{a} u \bar{u} \gamma^{\mu} \gamma_{5} t^{a} u\right), \\
Q_{V}^{s}=\left(\bar{u} \gamma_{\mu} u \bar{u} \gamma^{\mu} u\right), & Q_{A}^{s}=\left(\bar{u} \gamma_{\mu} \gamma_{5} u \bar{u} \gamma^{\mu} \gamma_{5} u\right) .
\end{array}
$$

Unfortunately, to our knowledge, for the flavour-diagonal correlators, the next-to-leading order corrections to the dimension- 6 four-quark operators are not available. The next important difference to the non-diagonal case is the fact that the two current-current operators $Q_{-}^{o, s}$ are not anymore closed under renormalisation, but all ten operators including the eight penguin operators are required. Also, now all operators are linearly independent. Hence, the complete set reads $Q_{-}^{\text {diag }} \equiv\left(Q_{-}^{o}, Q_{-}^{s}, Q_{3}, Q_{4}, Q_{5}, Q_{6}, Q_{7}, Q_{8}, Q_{9}, Q_{10}\right)$. For 
this set of operators, the leading-order anomalous dimension matrix is found to be

$$
\hat{\gamma}_{Q_{-}^{\text {diag }}}^{(1)}=\left(\begin{array}{cccccc}
-\frac{3 N_{c}}{2}+\frac{3}{N_{c}} & -\frac{3 C_{F}}{2 N_{c}} & \frac{2}{3} & 0 & 0 & \\
-3 & 0 & 0 & 0 & 0 & \\
0 & 0 & \frac{N_{f}}{3}-\frac{3 N_{c}}{4}-\frac{1}{3 N_{c}} & \frac{3 N_{c}}{4}-\frac{3}{N_{c}} & 0 & \\
0 & 0 & \frac{3 N_{c}}{4}-\frac{10}{3 N_{c}} & -\frac{3 N_{c}}{4} & \frac{3 C_{F}}{2 N_{c}} & \\
0 & 0 & \frac{2}{3} & 3 & 0 & \\
0 & 0 & \frac{11}{3} & 0 & 0 & \\
0 & 0 & 0 & 0 & 0 & \\
0 & 0 & 0 & 0 & 0 & \\
0 & 0 & 0 & 0 & 0 & \\
0 & 0 & 0 & 0 & 0 & \\
& 0 & 0 & 0 & 0 & 0 \\
& 0 & 0 & 0 & 0 & 0 \\
& \frac{3 C_{F}}{2 N_{c}} & \frac{1}{3} & 0 & 0 & 0 \\
& 0 & 0 & 0 & 0 & 0 \\
& 0 & 0 & 0 & 0 & 0 \\
& 0 & 0 & 0 & 0 & 0 \\
& 0 & \frac{2 N_{f}}{3}-\frac{3 N_{c}}{4}-\frac{1}{3 N_{c}} & \frac{3 N_{c}}{4}-\frac{3}{N_{c}} & 0 & \frac{3 C_{F}}{2 N_{c}} \\
& 0 & \frac{2}{3} & -\frac{3 N_{c}}{4} & \frac{3 C_{F}}{2 N_{c}} & 0 \\
& 0 & \frac{11}{3} & 0 & 0 & 0 \\
& 0 & 0 & 0 & 0
\end{array}\right) .
$$

For the flavour-diagonal $V+A$ correlator, the two current-current operators $Q_{+}^{o}$ and $Q_{+}^{s}$ are directly linearly related via Fierz transformations, with the relation being given by

$$
Q_{+}^{o}=\frac{1}{2}\left(1-\frac{1}{N_{c}}\right) Q_{+}^{s} .
$$

Choosing to remove the $Q_{+}^{s}$ operator, like in the non-diagonal case, we are again left we a set of nine operators, consisting of $Q_{+}^{\text {diag }} \equiv\left(Q_{+}^{o}, Q_{3}, Q_{4}, Q_{5}, Q_{6}, Q_{7}, Q_{8}, Q_{9}, Q_{10}\right)$. The corresponding leading-order anomalous dimension matrix is then found to be

$$
\begin{aligned}
& \hat{\gamma}_{Q_{+}^{\text {diag }}}^{(1)}=\left(\begin{array}{cccc}
\frac{3}{2}-\frac{3}{2 N_{c}} & \frac{2}{3}-\frac{2}{3 N_{c}} & 0 & 0 \\
0 & \frac{N_{f}}{3}-\frac{3 N_{c}}{4}-\frac{1}{3 N_{c}} & \frac{3 N_{c}}{4}-\frac{3}{N_{c}} & 0 \\
0 & \frac{3 N_{c}}{4}-\frac{10}{3 N_{c}} & -\frac{3 N_{c}}{4} & \frac{3 C_{F}}{2 N_{c}} \\
0 & \frac{2}{3} & 3 & 0 \\
0 & \frac{11}{3} & 0 & 0 \\
0 & 0 & 0 & 0 \\
0 & 0 & 0 & 0 \\
0 & 0 & 0 & 0 \\
0 & 0 & 0 & 0
\end{array}\right. \\
& \left.\begin{array}{ccccc}
0 & 0 & 0 & 0 & 0 \\
\frac{3 C_{F}}{2 N_{c}} & \frac{1}{3} & 0 & 0 & 0 \\
0 & 0 & 0 & 0 & 0 \\
0 & 0 & 0 & 0 & 0 \\
0 & 0 & 0 & 0 & 0 \\
0 & \frac{2 N_{f}}{3}-\frac{3 N_{c}}{4}-\frac{1}{3 N_{c}} & \frac{3 N_{c}}{4}-\frac{3}{N_{c}} & 0 & \frac{3 C_{F}}{2 N_{c}} \\
0 & \frac{3 N_{c}}{4}-\frac{10}{3 N_{c}} & -\frac{3 N_{c}}{4} & \frac{3 C_{F}}{2 N_{c}} & 0 \\
0 & \frac{2}{3} & 3 & 0 & 0 \\
0 & \frac{11}{3} & 0 & 0 & 0
\end{array}\right) .
\end{aligned}
$$


Let us again investigate the eigenvalues for the anomalous dimension matrices $\hat{\gamma}_{Q_{-}^{\text {diag }}}^{(1)}$ and $\hat{\gamma}_{Q_{+}^{\text {diag }}}^{(1)}$. Numerically, at $N_{c}=N_{f}=3$ and ordered in increasing value, they are found to be

$$
\begin{aligned}
& \vec{\gamma}_{D, Q_{-}^{\text {diag }}}^{(1)}=(-4,-3.611,-3.387,-1.878,-1.494,0.5,0.538,0.567,1.340,1.703), \\
& \vec{\gamma}_{D, Q_{+}^{\text {diag }}}^{(1)}=(-3.611,-3.387,-1.878,-1.494,0.538,0.567,1,1.340,1.703) .
\end{aligned}
$$

We first note, that the eigenvalues corresponding to $\hat{\gamma}_{Q_{+}^{\text {diag }}}^{(1)}$ are identical to the ones for $\hat{\gamma}_{Q_{+}}^{(1)}$ of eq. (2.32). This will have implications for the renormalon structure to be discussed in the next section. Furthermore, as can be inferred from the eigenvectors of eq. (B.2), the eigenvalue "1" corresponds to the current-current operators while the remaining ones involve penguin operators. The eigenvalues for $\hat{\gamma}_{Q_{-}^{\text {diag }}}^{(1)}$, on the other hand, include the ones from the non-diagonal current-current operators given in eq. (2.31) as well as again the ones involving the penguins.

\section{Renormalon structure of dimension-6 four-quark operators}

In this section, the perturbative ambiguities that are connected to the dimension- 6 fourquark OPE contributions, shall be investigated. The discussion closely follows section 3.3 of ref. [2] and section 5 of ref. [4].

Before investigating the renormalon structure, however, we have to briefly review the corresponding nomenclature. To begin, we introduce the Adler functions for non-diagonal vector and axial-vector correlators that are physical quantities in the sense that they are independent of renormalisation scale and scheme:

$$
D^{V / A}\left(Q^{2}\right) \equiv-Q^{2} \frac{\mathrm{d}}{\mathrm{d} Q^{2}} \Pi^{V / A}\left(Q^{2}\right)
$$

Next, we define the purely perturbative function $\widehat{D}_{0}\left(Q^{2}\right)$ through the relation

$$
D_{0}^{V / A}\left(Q^{2}\right) \equiv D_{0}\left(Q^{2}\right) \equiv \mathcal{N}\left[1+\widehat{D}_{0}\left(Q^{2}\right)\right]
$$

such that $\widehat{D}_{0}\left(Q^{2}\right)$ starts at order $\alpha_{s}$, and $\mathcal{N}=N_{c} /\left(12 \pi^{2}\right)$ is the common global normalisation. Because the perturbative part is identical for non-diagonal vector and axial-vector correlators, $\widehat{D}_{0}\left(Q^{2}\right)$ is the same in both cases.

Assuming a positive coupling $a_{Q}$, the Borel transform $B\left[\widehat{D}_{0}\right](u)$ of $\widehat{D}_{0}\left(a_{Q}\right)$ is defined by

$$
\widehat{D}_{0}\left(a_{Q}\right) \equiv \frac{2 \pi}{\beta_{1}} \int_{0}^{\infty} \mathrm{d} u \mathrm{e}^{-\frac{2 u}{\beta_{1} a} Q} B\left[\widehat{D}_{0}\right](u) .
$$

Because $D_{0}$ is dimensionless, its $Q^{2}$ dependence only arises via $a_{Q}$. Taylor expanding $B\left[\widehat{D}_{0}\right](u)$ and integrating term by term reproduces the perturbation series expansion of 
$\widehat{D}_{0}\left(a_{Q}\right)$. The integral on the right-hand side is, however, only well defined if $B\left[\widehat{D}_{0}\right](u)$ has no poles or cuts on the positive real $u$ axis which is not the case for the QCD Adler function. If poles and/or cuts, also termed infrared (IR) renormalon poles, are present on the integration interval, ambiguities appear since on has to specify how the poles are treated. (For example with the principal-value prescription.) It is generally assumed that those ambiguities should cancel against corresponding ambiguities in the OPE terms, in order to ensure that $D^{V / A}\left(Q^{2}\right)$ is physical.

From the generic structure of a term in the OPE, the general form of an infrared renormalon pole corresponding to an operator $O_{d}$ of dimension $d$ can be deduced, with the finding $[2,4]$

$$
B\left[D_{0}^{\mathrm{IR}}\right](u)=\frac{d_{p}^{\mathrm{IR}}}{(p-u)^{\kappa}}[1+\mathcal{O}(p-u)],
$$

where $d_{p}^{\mathrm{IR}}$ are non-perturbative normalisations (residua) of the renormalon poles that cannot be determined from renormalisation group arguments. On the other hand,

$$
p=\frac{d}{2}, \quad \kappa=1-\delta+2 p \frac{\beta_{2}}{\beta_{1}^{2}}-\frac{\gamma_{O_{d}}^{(1)}}{\beta_{1}} .
$$

Here, $\delta$ is the leading power in $a_{s}$ of the coefficient function, $\delta=1$ in our case of eqs. (2.3) and (2.4), and $\gamma_{O_{d}}^{(1)}$ is the leading order anomalous dimension of the operator $O_{d}$. Hence, the strength of the pole $\kappa$ (as well as all sub-leading terms) only depends on coefficients of the RG functions (and coefficient functions of the OPE contributions).

Before further discussing our particular case of the renormalon structure of dimension- 6 four-quark operators, we have to investigate which operators contribute to the perturbative ambiguity. To this end, we rewrite the current-current operators of eqs. (2.15) by means of Fierz transformations and separating the quark chiralities, which in the case of $Q_{-}^{o, s}$ leads to

$$
\begin{aligned}
Q_{-}^{o} & =-\frac{4 C_{F}}{N_{c}}\left(\bar{u}_{L} u_{R} \bar{d}_{R} d_{L}+(L \leftrightarrow R)\right)+\frac{4}{N_{c}}\left(\bar{u}_{L} t^{a} u_{R} \bar{d}_{R} t^{a} d_{L}+(L \leftrightarrow R)\right), \\
Q_{-}^{s} & =-\frac{4}{N_{c}}\left(\bar{u}_{L} u_{R} \bar{d}_{R} d_{L}+(L \leftrightarrow R)\right)-8\left(\bar{u}_{L} t^{a} u_{R} \bar{d}_{R} t^{a} d_{L}+(L \leftrightarrow R)\right) .
\end{aligned}
$$

From (4.6) and (4.7) it is seen that the operators $Q_{-}^{o, s}$ are order parameters of the $\mathrm{SU}\left(N_{f}\right)_{A}$ symmetry breaking and hence they cannot contribute to the perturbative ambiguity. This is also obvious from the fact that for non-diagonal quark currents in $V-A$, the purely perturbative contribution cancels and hence no related ambiguity can arise. This is different for the operators appearing in $V+A$, where rewriting the current-current operators $Q_{+}^{o, s}$ results in

$$
\begin{aligned}
Q_{+}^{o} & =\frac{2 C_{F}}{N_{c}}\left(\bar{u}_{L} \gamma_{\mu} u_{L} \bar{d}_{L} \gamma^{\mu} d_{L}+(L \rightarrow R)\right)-\frac{2}{N_{c}}\left(\bar{u}_{L} \gamma_{\mu} t^{a} u_{L} \bar{d}_{L} \gamma^{\mu} t^{a} d_{L}+(L \rightarrow R)\right), \\
Q_{+}^{s} & =\frac{2}{N_{c}}\left(\bar{u}_{L} \gamma_{\mu} u_{L} \bar{d}_{L} \gamma^{\mu} d_{L}+(L \rightarrow R)\right)+4\left(\bar{u}_{L} \gamma_{\mu} t^{a} u_{L} \bar{d}_{L} \gamma^{\mu} t^{a} d_{L}+(L \rightarrow R)\right) .
\end{aligned}
$$

Those two operators can and do have a perturbative ambiguity which is reflected in the perturbative series of the unit operator of the OPE for the $V+A$ correlator. The same holds true also for the penguin operators $Q_{3}$ to $Q_{10}$. 
Regarding the pole structure of IR renormalon poles corresponding to dimension- 6 operators in the OPE, the poles are located at $p=u=3$. Furthermore, at $N_{c}=3$ and for three quark flavours, the exponent $\kappa$ takes the value

$$
\kappa=\frac{64}{27}-\frac{2}{9} \gamma_{O_{6}}^{(1)} .
$$

The strongest singularity is assumed for the most negative eigenvalue in $\vec{\gamma}_{D, Q_{+}}^{(1)}$ of eq. (2.32), leading to $\kappa=3.173$, while the weakest pole corresponds to an exponent $\kappa=1.992$.

Let us briefly compare these findings with the large- $\beta_{0}$ approximation. ${ }^{4}$ This approximation can be obtained by considering the large- $N_{f}$ limit and then replacing $-N_{f} / 3 \rightarrow \beta_{1}$. In the anomalous dimension matrix of eq. (2.26) then only two entries, $-\beta_{1}$ and $-2 \beta_{1}$, for the operators $Q_{3}$ and $Q_{7}$, respectively, are left on the diagonal. Furthermore, the $\beta_{2}$ term in the exponent $\kappa$ is absent, so that $\kappa=1$ or 2 . This is precisely in line with the result of the large- $\beta_{0}$ approximation for the Adler function that at $u=3$ a linear and a quadratic pole is induced by dimension-6 operators [2]. Hence, it is observed that full QCD tends to make the $u=3 \mathrm{IR}$ renormalon poles stronger in comparison to the large- $N_{f}$ limit. This conclusion also remains true for the operators appearing in the case of flavour-diagonal correlators, because the eigenvalues of the anomalous dimension matrices are identical to the ones of the flavour non-diagonal case.

\section{Conclusions}

In this work, we revisited the calculation of one-loop anomalous dimension matrices of four-quark operators that enter vector and axial-vector correlators in QCD. We studied flavour diagonal and non-diagonal currents. Our results are given for an arbitrary number of quark colours, $N_{c}$, and flavours, $N_{f}$.

Explicit results for the anomalous dimension matrices of $V-A$ and $V+A$ flavour non-diagonal operators are given in eqs. (2.25) and (2.26). In the $V-A$ case, the set of two operators of eq. (2.15) is closed under renormalisation. The $V+A$ case requires the inclusion of 7 additional penguin-type operators to obtain a minimal closed set. We also presented the $\mathrm{SU}(2)$ flavour non-diagonal $V+A$ anomalous dimension matrix in eq. (2.33). Next, we investigated the anomalous dimension of flavour diagonal operators in $V-A$ and $V+A$ currents, given in eqs. (3.3) and (3.5). In the former, 8 penguin operators have to be added to the operator basis while in the latter 7 additional operators are sufficient.

The anomalous dimensions of some of these operators are related to renormalon singularities of the Borel transformed purely perturbative contribution of the QCD correlators. More specifically, part of the dimension- 6 four-quark operators are related to the subleading IR singularity located at $u=3$, to which an ambiguity in the Borel resummed series is associated. In order to investigate the specific structure of this singularity, it is instructive to work with diagonal bases of operators. In such bases, one isolates the combinations of operators that do not mix under renormalisation. The anomalous dimensions are

\footnotetext{
${ }^{4}$ For historical reasons, we speak about the "large- $\beta_{0}$ " approximation, although in the notation employed in this work, the leading coefficient of the $\beta$-function is termed $\beta_{1}$.
} 
simply the eigenvalues of the original anomalous dimension matrix. When a four-quark operator entails an IR singularity in the Borel transformed perturbative series, the respective eigenvalue is related to the strength of the IR singularity at $u=3$ as given by eq. (4.5).

The operators in the $V-A$ case do not have an associated ambiguity, since they are order parameters of the $\mathrm{SU}\left(N_{f}\right)_{A}$ symmetry breaking. The chiral structure of the $V+A$ operators indicates that they have renormalon singularities associated with them. Several singularities appear, one for each operator combination. The most negative eigenvalue yields the strongest singularity. In the $V+A$ non-diagonal case the corresponding exponent is $\kappa=3.173$. In comparison with the large- $N_{f}$ limit of QCD, in which one has a simple and a quadratic pole related to two dimension- 6 operators, the singularity in full QCD is stronger.

Finally, we should comment on the implications of our results to the Borel models of the Adler function that have been used in the discussion of the RG improvement of the perturbative series in hadronic $\tau$ decays $[4,5]$. As discussed in [5], the impact of the sub-leading IR singularity is limited since the Borel transform is dominated by the leading IR singularity, associated with the gluon condensate. Nevertheless, the results of the present work allow for a refined modelling of the IR singularity at $u=3$. However, the numerical value of the strength of the strongest IR singularity associated with dimension6 four-quark operators is rather close to the one already employed in $[4,5]$. The Borel transformed series modelled in this way is not altered in any significant way. Therefore, even taking into account the findings of the present study, the conclusions of refs. $[4,5]$ remain valid.

\section{Acknowledgments}

Interesting discussions with Martin Beneke, Antonio Pineda, and Santi Peris are gratefully acknowledged. We also thank Martin Beneke for carefully reading the manuscript. DB thanks the hospitality of Universitat Autònoma de Barcelona. This work has been supported in part by the Spanish Consolider-Ingenio 2010 Programme CPAN (Grant number CSD2007-00042), by MINECO Grant numbers CICYT-FEDER-FPA2011-25948 and CICYT-FEDER-FPA2014-55613-P, by the Severo Ochoa excellence program of MINECO under Grant number SO-2012-0234, and by Secretaria d'Universitats i Recerca del Departament d'Economia i Coneixement de la Generalitat de Catalunya under Grant number 2014 SGR 1450. The work of DB received support from the São Paulo Research Foundation (Fapesp) grant 14/50683-0.

\section{A Anomalous dimensions of four-quark operators}

In this appendix, we present a generalisation of the results of reference [7] to an arbitrary number $N_{c}$ of colour degrees of freedom. In [7], the leading order anomalous dimension matrix of a complete set of local spin-zero four-quark operators without derivatives was calculated in the case of three quark flavours. 
The complete basis consists of 45 four-quark operators which in reference [7] were chosen as follows: with respect to the Dirac-structure, there are five types of operators, namely, scalar, pseudoscalar, vector, axial vector and tensor. They can be expressed as

$$
\bar{u} \Gamma u \bar{d} \Gamma d=\left(\bar{u} u \bar{d} d, \bar{u} \gamma_{5} u \bar{d} \gamma_{5} d, \bar{u} \gamma_{\mu} u \bar{d} \gamma^{\mu} d, \bar{u} \gamma_{\mu} \gamma_{5} u \bar{d} \gamma^{\mu} \gamma_{5} d, \bar{u} \sigma_{\mu \nu} u \bar{d} \sigma^{\mu \nu} d\right)
$$

in the $\bar{u} u \bar{d} d$ flavour case. Employing this notation, the complete basis $O$ of operators can be chosen to be:

$$
\begin{array}{r}
O \equiv(\bar{u} \Gamma u \bar{u} \Gamma u, \bar{d} \Gamma d \bar{d} \Gamma d, \bar{s} \Gamma s \bar{s} \Gamma s, \bar{u} \Gamma u \bar{d} \Gamma d, \bar{u} \Gamma u \bar{s} \Gamma s, \bar{d} \Gamma d \bar{s} \Gamma s \\
\left.\bar{u} \Gamma t^{a} u \bar{d} \Gamma t^{a} d, \bar{u} \Gamma t^{a} u \bar{s} \Gamma t^{a} s, \bar{d} \Gamma t^{a} d \bar{s} \Gamma t^{a} s\right)
\end{array}
$$

In this basis, the leading order anomalous dimension matrix takes the form

$$
\gamma_{O}^{(1)}=\left(\begin{array}{ccccccccc}
A & 0 & 0 & 0 & 0 & 0 & B & B & 0 \\
0 & A & 0 & 0 & 0 & 0 & B & 0 & B \\
0 & 0 & A & 0 & 0 & 0 & 0 & B & B \\
0 & 0 & 0 & C & 0 & 0 & D & 0 & 0 \\
0 & 0 & 0 & 0 & C & 0 & 0 & D & 0 \\
0 & 0 & 0 & 0 & 0 & C & 0 & 0 & D \\
E & E & 0 & F & 0 & 0 & G & H & H \\
E & 0 & E & 0 & F & 0 & H & G & H \\
0 & E & E & 0 & 0 & F & H & H & G
\end{array}\right)
$$

The submatrices are given by:

$$
\begin{aligned}
& A=\left(\begin{array}{ccccc}
\frac{11}{12}-3 C_{F} & \frac{7}{12} & -\frac{1}{12}+\frac{1}{6 N_{c}} & -\frac{1}{12} & -\frac{1}{8}+\frac{1}{4 N_{c}} \\
\frac{7}{12} & \frac{11}{12}-3 C_{F} & \frac{1}{12}-\frac{1}{6 N_{c}} & \frac{1}{12} & -\frac{1}{8}+\frac{1}{4 N_{c}} \\
\frac{7}{6} & -\frac{7}{6} & \frac{11}{12}-\frac{1}{3 N_{c}} & \frac{11}{12}-\frac{3}{2 N_{c}} & 0 \\
-\frac{11}{6} & \frac{11}{6} & \frac{11}{12}-\frac{11}{6 N_{c}} & \frac{11}{12} & 0 \\
3+\frac{6}{N_{c}} & 3+\frac{6}{N_{c}} & 0 & 0 & \frac{3}{2}+C_{F}
\end{array}\right), \\
& B=\left(\begin{array}{ccccc}
0 & 0 & -\frac{1}{3} & 0 & 0 \\
0 & 0 & \frac{1}{3} & 0 & 0 \\
0 & 0 & \frac{2}{3} & 0 & 0 \\
0 & 0 & \frac{2}{3} & 0 & 0 \\
0 & 0 & 0 & 0 & 0
\end{array}\right), \quad C=\left(\begin{array}{ccccc}
-3 C_{F} & 0 & 0 & 0 & 0 \\
0 & -3 C_{F} & 0 & 0 & 0 \\
0 & 0 & 0 & 0 & 0 \\
0 & 0 & 0 & 0 & 0 \\
0 & 0 & 0 & 0 & C_{F}
\end{array}\right), \\
& D=\left(\begin{array}{ccccc}
0 & 0 & 0 & 0 & -\frac{1}{2} \\
0 & 0 & 0 & 0 & -\frac{1}{2} \\
0 & 0 & 0 & 3 & 0 \\
0 & 0 & 3 & 0 & 0 \\
-12 & -12 & 0 & 0 & 0
\end{array}\right), \quad E=\left(\begin{array}{ccccc}
0 & 0 & 0 & 0 & 0 \\
0 & 0 & 0 & 0 & 0 \\
-\frac{1}{6} & \frac{1}{6} & \frac{1}{12}-\frac{1}{6 N_{c}} & \frac{1}{12} & 0 \\
0 & 0 & 0 & 0 & 0 \\
0 & 0 & 0 & 0 & 0
\end{array}\right),
\end{aligned}
$$




$$
\begin{aligned}
G & =\left(\begin{array}{cccccc}
\frac{3}{2 N_{c}} & 0 & 0 & 0 & -\frac{N_{c}}{8}+\frac{1}{2 N_{c}} \\
0 & \frac{3}{2 N_{c}} & 0 & 0 & -\frac{N_{c}}{8}+\frac{1}{2 N_{c}} \\
0 & 0 & -\frac{3 N_{c}}{4}+\frac{2}{3} \frac{3 N_{c}}{4}-\frac{3}{N_{c}} & 0 \\
0 & 0 & \frac{3 N_{c}}{4}-\frac{3}{N_{c}} & -\frac{3 N_{c}}{4} & 0 \\
-3 & N_{c}+\frac{12}{N_{c}}-3 N_{c}+\frac{12}{N_{c}} & 0 & 0 & C_{F}-\frac{3 N_{c}}{2}
\end{array}\right), \\
H & =\left(\begin{array}{lllll}
0 & 0 & 0 & 0 & 0 \\
0 & 0 & 0 & 0 & 0 \\
0 & 0 & \frac{1}{3} & 0 & 0 \\
0 & 0 & 0 & 0 & 0 \\
0 & 0 & 0 & 0 & 0
\end{array}\right) .
\end{aligned}
$$

In contrast to ref. [7], here, the matrices $A, C$ and $G$ already include the quark selfenergy contributions depicted in figure 1c) of [7], such that they are gauge independent. (The corresponding matrices of [7] were given in the Feynman gauge without self-energy contribution.)

This basis of operators is particularly handy to derive the following relation between the 10 operators of the redundant basis of non-diagonal currents given in eqs. (2.5) to (2.15). We find

$$
Q_{5}=\frac{N_{c}}{N_{c}-1}\left(2 Q_{+}^{o}+2 Q_{3}+2 Q_{4}-Q_{7}-Q_{8}\right)-Q_{+}^{s}-Q_{6}+\frac{1}{2}\left(Q_{9}+Q_{10}\right) .
$$

\section{B Eigenvectors of anomalous dimension matrices}

Here, we present the eigenvectors of the non-diagonal anomalous dimension matrices of eqs. (2.25) and (2.26). The coefficients of the eigenvectors are displayed as the columns of the matrices $\hat{V}$ that diagonalise the bases, as defined in eq. (2.27). The results are given for $N_{c}=N_{f}=3$. For the $V-A$ case one finds

$$
\hat{V}_{Q_{-}}=\left(\begin{array}{rr}
\frac{4}{3} & -\frac{1}{6} \\
1 & 1
\end{array}\right) .
$$

The matrix $\hat{V}_{Q_{+}}$, for the $V+A$ case, reads

$$
\hat{V}_{Q_{+}}=\left(\begin{array}{rrrrrrrrr}
-0.059 & -0.028 & 0.472 & -0.093 & 0.045 & -0.015 & 0.316 & 0.081 & -0.020 \\
0.145 & 0.066 & -0.664 & 0.100 & 0.082 & -0.027 & 0.949 & 0.338 & -0.117 \\
-0.519 & -0.209 & -0.254 & 0.191 & -0.138 & 0.043 & 0 & 0.313 & -0.208 \\
0.654 & 0.386 & -0.159 & 0.077 & 0.292 & -0.115 & 0 & 0.219 & -0.105 \\
0.527 & 0.226 & 0.496 & -0.469 & -0.942 & 0.275 & 0 & 0.856 & -0.447 \\
0 & -0.314 & 0 & 0.287 & 0 & 0.064 & 0 & 0 & -0.312 \\
0 & 0.578 & 0 & 0.115 & 0 & -0.173 & 0 & 0 & -0.157 \\
0 & -0.450 & 0 & -0.359 & 0 & -0.839 & 0 & 0 & -0.399 \\
0 & 0.340 & 0 & -0.703 & 0 & 0.413 & 0 & 0 & -0.671
\end{array}\right) .
$$


Open Access. This article is distributed under the terms of the Creative Commons Attribution License (CC-BY 4.0), which permits any use, distribution and reproduction in any medium, provided the original author(s) and source are credited.

\section{References}

[1] G. 't Hooft, Can we make sense out of Quantum Chromodynamics?, Subnucl. Ser. 15 (1979) 943 [INSPIRE].

[2] M. Beneke, Renormalons, Phys. Rept. 317 (1999) 1 [hep-ph/9807443] [InSPIRE].

[3] E. Braaten, S. Narison and A. Pich, QCD analysis of the tau hadronic width, Nucl. Phys. B 373 (1992) 581 [INSPIRE].

[4] M. Beneke and M. Jamin, $\alpha_{s}$ and the $\tau$ hadronic width: fixed-order, contour-improved and higher-order perturbation theory, JHEP 09 (2008) 044 [arXiv:0806.3156] [INSPIRE].

[5] M. Beneke, D. Boito and M. Jamin, Perturbative expansion of $\tau$ hadronic spectral function moments and $\alpha_{s}$ extractions, JHEP 01 (2013) 125 [arXiv: 1210.8038] [INSPIRE].

[6] R.D.C. Miller and B.H.J. McKellar, Anomalous dimension matrices of four quark operators, Phys. Rev. D 28 (1983) 844 [InSPIRE].

[7] M. Jamin and M. Kremer, Anomalous dimensions of spin-0 four quark operators without derivatives, Nucl. Phys. B 277 (1986) 349 [InSPIRE].

[8] L.E. Adam and K.G. Chetyrkin, Renormalization of four quark operators and QCD sum rules, Phys. Lett. B 329 (1994) 129 [hep-ph/9404331] [INSPIRE].

[9] L.V. Lanin, V.P. Spiridonov and K.G. Chetyrkin, Contribution of four quark condensates to sum rules for $\rho$ and $A_{1}$ mesons (in Russian), Yad. Fiz. 44 (1986) 1372 [INSPIRE].

[10] M. Beneke, V.M. Braun and N. Kivel, Large order behavior due to ultraviolet renormalons in QCD, Phys. Lett. B 404 (1997) 315 [hep-ph/9703389] [INSPIRE].

[11] S. Descotes-Genon and B. Malaescu, A note on renormalon models for the determination of $\alpha_{s}\left(M_{\tau}\right)$, arXiv:1002.2968 [INSPIRE].

[12] A.J. Buras, Asymptotic freedom in deep inelastic processes in the leading order and beyond, Rev. Mod. Phys. 52 (1980) 199 [inSPIRE].

[13] A.J. Buras, M. Jamin, M.E. Lautenbacher and P.H. Weisz, Effective Hamiltonians for $\Delta S=1$ and $\Delta B=1$ non-leptonic decays beyond the leading logarithmic approximation, Nucl. Phys. B 370 (1992) 69 [inSPIRE]. 